\title{
Sintomas depressivos e fatores associados em puérperas de um hospital-escola em Cuiabá/MT
}

\author{
Alessandra Messias de Almeida* \\ Rosangela Kátia Sanches Mazzorana Ribeiro**
}

\begin{abstract}
Resumo
A Depressão Pós-Parto (DPP) é um problema de saúde pública mundial. Os fatores de risco para seu desenvolvimento podem variar considerando-se aspectos psicossociais. Este estudo objetivou identificar sintomas depressivos e fatores associados em puérperas no Hospital Universitário Júlio Muller (HUJM), na cidade de Cuiabá - MT. Participaram 30 puérperas, entre 18 anos e 39 anos ( $M=26,96$ anos). Como instrumentos foram utilizados entrevista semiestruturada e questionário PHQ-9. Os dados foram analisados por meio de estatística descritiva e do modelo de regressão linear simples (Teste F), que correlacionou dados da entrevista e escores do PHQ-9. Como resultados, constatou-se que a maioria eram multíparas, com baixa escolaridade e em relacionamento estável. Apresentaram sinais de risco para o desenvolvimento da DPP metade das participantes, sendo o estresse gestacional o principal fator associado. Os resultados evidenciam a importância da identificação precoce dos sintomas depressivos e ressaltam a importância da avaliação psicológica para tal identificação na área da saúde.
\end{abstract}

Palavras-chave: Depressão pós-parto, Avaliação Psicológica, PQH-9

\begin{abstract}
Postpartum depression (PPD) is a worldwide public health problem. The risk factors for its development can vary considering psychosocial aspects. This study aimed to identify depressive symptoms and associated factors in puerperal women at the Júlio Muller University Hospital (HUJM), in the city of Cuiabá - MT. 30 puerperal women participated, between 18 years and 39 years $(M=26.96$ years). As instruments, semi-structured interviews and a PHQ-9 questionnaire were used. The data were analyzed using descriptive statistics and the simple linear regression model (Test F), which correlated interview data and PHQ-9 scores. As a result, it was found that the majority were multiparous, with low education and in a stable relationship. Half of the participants showed signs of risk for the development of PPD, with gestational stress being the main associated factor. The results show the importance of early identification of depressive symptoms and emphasize the importance of psychological assessment for such identification in the health area.
\end{abstract}

Key-words: Postpartum depression, Psychological Assessment, PHQ-9

* Psicóloga pela Universidade de Cuiabá e Mestre em Psicologia - UFMP

** Psicóloga pela Universidade de Cuiabá, Mestre em Psicologia - UCRGS e Doutorado em Psiquiatria e Psicologia Médica - UNIFESP 


\section{Introdução}

O ciclo gravídico-puerperal é um período de risco elevado para o desenvolvimento da depressão e de sintomas, em razão de alterações hormonais, físicas e emocionais vivenciadas pela mulher (Hartmann, Mendoza-Sassi, \& Cesar, 2017; Lobato, Moraes, \& Reichenheim, 2011; Marques, Silva, Lima, Nunes, Ferreira \& Fernandes, 2016; Oliveira, Teixeira, Costa, Alencar, Rodrigues, Lima \& Chaves, 2019). Apesar de ser um momento esperado por muitas mulheres, o nascimento do bebê destaca-se como de maior vulnerabilidade emocional e risco potencial para o desenvolvimento de transtornos psicológicos como a depressão (Theme-Filha, Baldisserotto, Fraga, Ayers, Gama \& Leal 2016).

A Depressão Pós-Parto (DPP), também denominada depressão puerperal é considerada mundialmente um problema de saúde pública (Arrais, Araújo, \& Schiavo, 2018; Ferreira, Silva, Guerra, Silva, \& Rosário, 2018; Oliveira, Matão, Diego, Resende, \& Cleusa, 2020; OPAS/ OMS, 2017; Theme-Filha, et al., 2016). Caracteriza-se por tristeza persistente e perda de interesses por atividades habituais, e está entre as principais causas de problemas de saúde em todo o mundo (OPAS/OMS, 2017).

De acordo com a Associação Americana de Psiquiatria (American Psychiatric Association, 2014) constitui-se como Transtorno Depressivo, de origem multifatorial, cujos sintomas podem ter início durante a gestação ou até quatro semanas após o parto. Segundo a Organização Mundial de Saúde (OPAS/OMS, 2018) a depressão está entre as 20 principais doenças que podem causar incapacidade na população e sua prevalência é maior entre pessoas de 15 a 59 anos, com risco aumentado entre as mulheres.

Apesar de ser uma temática amplamente discutida na literatura científica, com estudos nacionais e internacionais, "a adoção de diferentes escalas de triagem para o diagnóstico do quadro, aplicadas em momentos diversos, tem por consequência uma variabilidade dos resultados encontrados pelas pesquisas" (Hildebrandt, 2013, p. 10). A prevalência da DPP no contexto brasileiro sugere variabilidade em suas estimativas. Para Hildebrandt (2013) o percentual é de 7,2 a 43\%; Theme-Filha, et al. (2016) de 25\%; Hartmann, Mendoza-Sassi, e Cesar (2017) de 30 a $40 \%$ e Oliveira, Matão, Diego, Resende, e Cleusa (2020) estimam de 12 a 39,4\%.

A Escala de Depressão Pós-Parto de Edimburgo (EPDS) é apontada como o principal instrumento utilizado em estudos brasileiros para a identificação dos sintomas (Hildebrandt, 2013; Arrais \& Araújo, 2017). Em contrapartida, estudos internacionais apontam o questionário PHQ-9 como um importante instrumento no rastreio de sintomas depressivos. Yawn, Pace, Wollan, Bertram, Kurland, Graham e Dietrich, 2009 realizaram estudo comparativo entre o questionário PHQ-9 e a EPDS, avaliando a efetividade de ambos os instrumentos na identificação dos sintomas e constataram elevada concordância entre os resultados (83\%). Outro estudo americano de Harrington, Hosseinipour, Maliwichi, Phulusa, Jumbe, Wallie, Gaynes, Maselko, Miller e Pence (2018) constatou concordância ainda mais elevada $(96 \%)$.

De acordo com Arrais e Araújo (2017, p. 830) "fatores de risco são eventos ou situações já estabelecidas são propícias ao surgimento de problemas físicos, psicológicos e sociais". Tais fatores podem variar considerando-se o contexto sociocultural da mulher (Marques, et al., 2016). Estudo de Arrais, Araújo e Schiavo (2018) apontou a falta de planejamento gestacional e falta de apoio familiar como principais fatores de risco para o desenvolvimento da DPP. Sugerem que o pré-natal psicológico, associado a fatores de proteção podem constituir medida preventiva para a saúde mental materna. O Ministério da Saúde (MS, 2019) ressalta o histórico de transtornos mentais; a falta de apoio familiar, do parceiro e amigos, estresse, problemas financeiros ou familiares; falta de planejamento gestacional; limitações físicas anteriores a gestação; histórico familiar de transtornos mentais; histórico de desordem disfórica pré-menstrual e violência doméstica, como principais fatores de risco para a DPP.

\section{Objetivo:}

Este estudo teve como objetivo identificar sintomas depressivos e fatores associados em puérperas atendidas no Hospital Universitário Júlio Muller (HUJM) - Cuiabá/ Mato Grosso.

\section{Método}

Trata-se de uma pesquisa descritiva, correlacional, de corte longitudinal e abordagem quantitativa.

\section{Participantes}

A amostra foi constituída por conveniência, com 30 puérperas, na faixa etária entre 18 e 39 anos $(M=26,96$ anos e $\mathrm{DP}=5,8)$, recém paridas e sem diagnóstico de transtornos pré-existentes. Eram moradoras de municípios do estado de Mato Grosso, atendidas na enfermaria de Ginecologia e Obstetrícia do Hospital Universitário Júlio Muller (HUJM), da cidade de Cuiabá (MT). 


\section{Instrumentos}

Nesta pesquisa foram utilizados dois instrumentos, designadamente uma entrevista semiestruturada (elaborada pelas pesquisadoras), utilizada para realizar a caracterização geral das participantes e de suas condições obstétricas, e o questionário PHQ-9 para rastrear sinais de risco quanto a depressão, adotando-se um ponto de corte $\geq 9$ (Santos, Tavares, Almeida, Silva, Tams, Patella \& Matijasevich, 2013). O PHQ-9 é um instrumento, que avalia depressão maior, pelos sintomas interesse e prazer; perspectiva; sono; fadiga; apetite; fracasso; concentração; lentidão ou agitação; pensamentos autodestrutivos e alteração de rotina.

\section{Local da coleta}

A coleta de dados foi realizada no Hospital Universitário Júlio Muller, localizado na cidade de Cuiabá/ Mato Grosso. O hospital foi fundado em 1984, vinculado a Universidade Federal de Mato Grosso, tornando-se hospital escola, que abrange a capital, Baixada Cuiabana e 14 municípios do estado. Atualmente é referência para acompanhamento de gestações e partos de alto risco. Embora tenha se tornado referência na área de ginecologia e obstetrícia, o hospital não tem programas específicos voltados a avaliação e acompanhamento da saúde mental materna.

\section{Procedimentos}

A pesquisa foi aprovada pelo Comitê de Ética em Pesquisa do Hospital Universitário Júlio Muller, sob o protocolo $\mathrm{n}^{\circ}$. CAEE 98684118.8.0000.5541, seguindo as orientações da Resolução CNS/Ministério da Saúde, $n^{\circ}$ 466, de 12 de dezembro de 2012, referente aos cuidados éticos na pesquisa com seres humanos.

Para as participantes que consentiram sua participação por meio do Termo de Consentimento Livre e Esclarecido, a coleta de dados foi realizada de modo individual, na enfermaria do referido hospital.

\section{Análise de dados}

Após a coleta de dados, as informações sociodemográficas e do PHQ-9 foram tabuladas na planilha do software Microsoft Excel 2013®. Foi feita análise exploratória, e descrição da média, desvio-padrão e medianas (mínimo-máximo) da entrevista. Quanto ao questionário PHQ-9 foi feita mensuração dos escores individuais e levantamento de percentual de participantes com risco aumentado para o desenvolvimento da depressão. A fim de identificar os fatores associados a DPP, utilizou-se o teste $\mathrm{F}$ de regressão linear simples, cruzando os dados sociodemográficos (obtidos por meio da entrevista) e escores do PHQ-9.

\section{Resultados}

Quanto aos resultados da pesquisa, a tabela 1 apresenta a caracterização sociodemográfica das participantes e condições obstétricas, a saber: escolaridade, estado civil, tratamento psicológico anterior, planejamento gestacional, risco gestacional, histórico de abortos, situação estressante na gestação, tipo de parto e convivência com o pai do bebê.

Tabela 1 - Caracterização sociodemográfica e condições obstétricas das puérperas do Hospital Júlio Muller de Cuiabá/MT

\begin{tabular}{|c|c|c|}
\hline Variável & $\mathrm{N}$ & $\%$ \\
\hline \multicolumn{3}{|l|}{$\overline{\text { Idade }}$} \\
\hline 18 a 28 anos & 20 & 66,7 \\
\hline 29 a 39 anos & 10 & 33,3 \\
\hline \multicolumn{3}{|l|}{ Escolaridade } \\
\hline Ensino fundamental incompleto & 3 & 10,0 \\
\hline Ensino fundamental completo & 3 & 10,0 \\
\hline Ensino médio incompleto & 12 & 40,0 \\
\hline Ensino médio completo & 6 & 20,0 \\
\hline Ensino superior incompleto & 4 & 13,3 \\
\hline Ensino superior completo & 2 & 6,7 \\
\hline \multicolumn{3}{|l|}{ Estado Civil } \\
\hline Solteira & 4 & 13,3 \\
\hline União estável & 15 & 50,0 \\
\hline Casada & 8 & 26,7 \\
\hline Separada & 3 & 10,0 \\
\hline \multicolumn{3}{|l|}{ Tratamento psicológico anterior } \\
\hline Sim & 6 & 20,0 \\
\hline Não & 24 & 80,0 \\
\hline \multicolumn{3}{|l|}{ Planejamento gestacional } \\
\hline Sim & 8 & 26,7 \\
\hline Não & 22 & 73,3 \\
\hline \multicolumn{3}{|l|}{ Risco gestacional } \\
\hline Sim & 14 & 46,7 \\
\hline Não & 16 & 53,3 \\
\hline \multicolumn{3}{|l|}{ Histórico de abortos } \\
\hline Sim & 5 & 16,7 \\
\hline Não & 25 & 83,3 \\
\hline \multicolumn{3}{|l|}{ Situação estressante na gestação } \\
\hline $\operatorname{sim}$ & 16 & 53,3 \\
\hline Não & 14 & 46,7 \\
\hline \multicolumn{3}{|l|}{ Tipo do parto } \\
\hline Normal & 22 & 73,3 \\
\hline Cesáreo & 8 & 26,7 \\
\hline \multicolumn{3}{|l|}{ Convivência com o pai do bebê } \\
\hline Sim & 22 & 73,3 \\
\hline Não & 8 & 26,7 \\
\hline
\end{tabular}




\section{ALESSANDRA MESSIAS DE ALMEIDA \\ ROSANGELA KÁTIA SANCHES MAZZORANA RIBEIRO}

Em relação a idade das participantes, a idade das participantes variou entre 18 anos e 39 anos $(M=26,96$ anos / DP $=5,8)$, sendo que a maioria $(66,7 \%)$ tinham até 28 anos. Quanto à escolaridade, 60\% $(n=18)$ tinham ensino médio completo/incompleto. Quanto ao estado civil a maioria declarou vivenciar algum tipo de relacionamento, sendo 76,7\% ( $\mathrm{n}=23)$ em união estável/casadas, $13,3 \%(n=4)$ solteiras e 10\% $(n=3)$ separadas. Quanto a tratamento psicológico anterior, $80 \%(\mathrm{n}=24)$ informaram não ter realizado.

Em relação as condições obstétricas, a maioria $73 \%(n=22)$ eram multíparas e o mesmo percentual informaram não ter planejado a gestação. Quanto ao risco gestacional, observou-se que a maioria 53,3\% ( $\mathrm{n}=$ 16) transcorreu de forma habitual, sem riscos ou complicações para a mãe ou o bebê, e apenas $16,7 \%(n=5)$ relataram histórico de aborto. O tipo de parto predominante foi o normal $73,3 \%(n=22)$ e o mesmo percentual informaram ter contato ou convivência com o pai do bebê. A maioria das participantes 53,3\% $(n=16)$ informaram ter vivenciado algum tipo de situação estressante ao longo da gestação.

Os motivos estressores declarados pelas puérperas serão apresentados na tabela 2.

Tabela 2 - Motivos estressores

\begin{tabular}{llr}
\hline Variável & N & $\%$ \\
\hline Conflito conjugal & 4 & $25,0 \%$ \\
Condições financeiras & 2 & $12,5 \%$ \\
Queda na rua & 1 & $6,3 \%$ \\
Incerteza da paternidade & 2 & $12,5 \%$ \\
Saúde do bebê & 3 & $18,8 \%$ \\
Lida com outros filhos & 3 & $18,8 \%$ \\
Separação conjugal & 1 & $6,3 \%$ \\
\hline
\end{tabular}

A tabela 2 demonstra os motivos estressores declarados pelas puérperas em relação ao estresse gestacional. O conflito conjugal, para25\% $(n=4)$ das participantes foi o principal motivo, seguido com o mesmo percentual por preocupação com a saúde do bebê e a lida com outros filhos 18,8\% $(n=3)$ para cada, preocupações financeiras e incerteza quanto a paternidade do bebê com 12,5\% ( $\mathrm{n}=$ 2) para cada, e queda em deslocamento na rua e separação conjugal também com mesmo percentual 6,3\% ( $\mathrm{n}=1)$.

Quanto aos escores das participantes no questionário PHQ-9, constatou-se que 50\% ( $\mathrm{M}=9,83$ / $\mathrm{DP}=$ $6,34)$ apresentaram sinais de risco para o desenvolvimento da DPP, considerando-se ponto de corte $\geq 9$ (Santos, et al., 2013). Os principais sintomas foram alterações do sono $(20 \%)$, seguidos de fadiga e alterações do apetite com mesmo percentual (16\%) cada.

Quanto a severidade dos sintomas os resultados serão apresentados na Tabela 3, a seguir.

Tabela 3 - Severidade dos sintomas no PHQ-9

\begin{tabular}{lrr}
\hline \multicolumn{1}{c}{ Gravidade } & \multicolumn{2}{c}{ PHQ-9 } \\
\hline & $\mathrm{N}$ & $\%$ \\
$0-4$ Nenhum ou mínimo & 8 & 26,7 \\
$5-9$ Leve & 7 & 23,3 \\
$10-14$ Moderado & 5 & 16,7 \\
15 - 19 Moderadamente severo & 8 & 26,7 \\
20 - 27 Severo & 2 & 6,7 \\
Geral & 30 & 100 \\
\hline
\end{tabular}

A Tabela 3, mostra que apenas 26,7\% $(n=8)$ não apresentaram riscos ou apenas riscos mínimos para o desenvolvimento da DPP. As demais participantes apresentaram sinais entre leve a severo, sendo 40\% ( $\mathrm{n}=$ 12) leves/moderados das participantes e $33,4 \%(n=10)$ moderadamente severos/severos.

Os resultados da correlação realizada pelo Teste $\mathrm{F}$ entre os dados sociodemográficos (obtidos por meio da entrevista) e indicador total do PHQ-9 serão apresentados na Tabela 4, a seguir.

Tabela 4 - Teste de correlação dos dados sociodemográficos e escores do PHQ-9

\begin{tabular}{lcrcc}
\hline Variável & Correlação & $\mathrm{R}^{2}$ & Estatística F & $\mathrm{p}$-valor \\
\hline Escolaridade & 0,01 & 0,0001 & 0,0019 & 0,9654 \\
Estado Civil & 0,16 & 0,0247 & 0,7106 & 0,4064 \\
Tratamento psicológico anterior & 0,03 & 0,0007 & 0,0200 & 0,8885 \\
Planejamento gestacional & 0,23 & 0,0509 & 1,5026 & 0,2305 \\
Risco gestacional & 0,10 & 0,0100 & 0,2830 & 0,5990 \\
Histórico de abortos & 0,09 & 0,0078 & 0,2209 & 0,6420 \\
Situação estressante na gestação & 0,43 & 0,1807 & 6,1756 & $0,0192^{*}$ \\
Tipo do parto & 0,26 & 0,0686 & 2,0628 & 0,1620 \\
Convivência com o pai do bebê & 0,01 & 0,0001 & 0,0018 & 0,9663 \\
\hline
\end{tabular}


A Tabela 4 indica que apenas a variável situação estressante na gestação $(p=0,0192)$ foi associada a sintomas depressivos, apresentando resultado estatisticamente significativo ao nível $\mathrm{p}<0,05$.

\section{Discussão}

Quanto a caracterização geral da amostra, os achados são semelhantes aos resultados de estudos nacionais (Hartmann, Mendoza-Sassi, \& Cesar, 2017; Marques et al., 2016; Oliveira et al., 2019), que apontaram mulheres jovens, com escolaridade no ensino médio (incompleto e completo), multíparas e vivenciando relacionamento estável e estudo de Hartmann, Mendoza-Sassi, e Cesar (2017) descrevem a multiparidade e escolaridade como fatores de risco ao desenvolvimento da DPP, que não foram associados neste estudo. Em relação a idade das puérperas, constatou-se média ligeiramente inferior (26,96 anos) a média da região Centro-Oeste divulgada pelo Ministério da Saúde (2009) com base na Pesquisa Nacional de Demografia (PNDS) realizada em 2006, que indicou média superior a 31 anos para mulheres em idade reprodutiva no Brasil, diferindo da amostra deste estudo.

A análise dos dados converge com outros estudos em relação a falta de planejamento gestacional das puérperas (Arrais \& Araújo, 2018; Marques et al., 2016; Oliveira et al., 2019), no entanto, não foi associada nesse estudo como fator de risco, divergindo de outras pesquisas que evidenciaram tal associação (Arrais \& Araújo, 2018; Krob, Godoy, Leite, \& Mori, 2017; Marques et al., 2016; Ministério da Saúde, 2019). Em contrapartida, Hartmann, Mendoza-Sassi, e Cesar (2017) destacam que o planejamento gestacional pode ser considerado um fator de proteção a DPP, pois reduzem em até $30 \%$ os riscos para o desenvolvimento da doença.

O tipo de parto predominante foi o normal $(73,3 \%)$. Tal achado pode estar relacionado a ênfase dada pelas políticas públicas de valorização a este tipo de parto na atualidade (MS, 2017). Estudo realizado por Biscegli, Silva, Romualdo, Oliveira, Silva e Solim (2017) constatou maior prevalência da DPP em mulheres que tiveram parto cesáreo. No entanto, nesse estudo, os resultados divergiram, apontando que apesar da maioria $(73,3 \%)$ ter tido parto normal, o percentual de puérperas com sintomas depressivos foi elevado (50\%), comparado a outros estudos (Hartmann, Mendoza-Sassi, \& Cesar, 2017; Hildebrandt, 2013; Oliveira et al. 2020; Theme-Filha, et al., 2016). No entanto, tal percentual reforça a necessidade quanto ao rastreio precoce dos sintomas, a fim de promover estratégias preventivas para a saúde mental materna, conforme
(Arrais \& Araújo 2017; Arrais, Araújo, \& Schiavo, 2018; Ferreira, Silva, Guerra, Silva, \& Rosário, 2018; Hartmann, Mendoza-Sassi, \& Cesar, 2017).

A análise de regressão linear simples associou o estresse gestacional como fator de risco para a DPP. Tal achado corrobora com estudo de Kliemann, Böing, e Crepaldi, (2017), que indicam a vivência de eventos estressores na gestação entre os principais fatores de risco. Ressalta-se a complexidade da etiologia depressiva no ciclo gravídico-puerperal, considerando a variedade de aspectos pessoais, fisiológicos, contextuais e relacionais que podem influenciar a condição psicológica materna Kliemann, Böing, e Crepaldi, (2017). Apesar da complexidade da DPP e do elevado percentual de puérperas com sinais de risco aumentado nesse estudo, o hospital em que a pesquisa foi realizada não tem programas de atenção à saúde mental materna. Nesse sentido, no âmbito da saúde materno-infantil, podem ser pensadas políticas públicas que promovam acolhimento, avaliação e orientação em todo o ciclo gravídico-puerperal, identificando sinais de risco precocemente.

A vivência de conflitos conjugais destacou-se como o principal estressor ao longo da gestação (25\%). Uma porcentagem um pouco menor (18,8\%) foi identificada em outros dois motivos, sendo a lida com outros filhos e preocupações com a saúde do bebê. Estudo de Arrais e Araújo (2017) corroboram com tal achado, apontando o estresse gestacional como fator de risco. Nesse sentido, Cunha, Santos, e Gonçalves (2012) destacam a importância do apoio social, seja da família, companheiro ou grupos de referência, como suporte psíquico salutar no ciclo gravídico-puerperal.

Acerca da rede de apoio, a maioria das participantes $(73,3 \%)$ informaram ter convivência ou contato com o pai do bebê. No entanto, não podemos afirmar que tal convivência represente de fato apoio social, considerando que este seja "um construto multidimensional envolvendo o conforto, a assistência e/ou informações que alguém recebe de contatos sociais formais ou informais" (Cardoso \& Vivian, 2017, p.44). Nesse sentido, não podemos afirmar que a convivência lhes garante troca e apoio de fato.

\section{Considerações finais}

A temática discutida nesse estudo é atual e relevante no campo da saúde mental materna. Ressalta-se que os resultados encontrados podem contribuir com demais áreas da saúde, no entanto, reforça-se a necessidade de estudos complementares que possam ampliar a compreensão dos fatores desencadeantes da DPP. 


\section{ALESSANDRA MESSIAS DE ALMEIDA \\ ROSANGELA KÁTIA SANCHES MAZZORANA RIBEIRO}

Considera-se que o rastreio precoce dos sinais de risco para o desenvolvimento da DPP, seja um aspecto importante a ser discutido por profissionais de saúde, principalmente com equipe multidisciplinar, prevenindo assim agravos a saúde mental materna. Nesse sentido, ressalta-se a importância da Avaliação Psicológica, para que ainda possam auxiliar na incrementação de programas de atenção as puérperas. Destaca-se ainda as contribuições para a Psicologia, principalmente no contexto hospitalar, para a compreensão dos aspectos que envolvem a DPP, considerando sua multifatorialidade.

\section{Referências bibliográficas}

American Psychiatric Association. (2014). Manual Diagnóstico e Estatítico de Transtornos Mentais - DSM V. Porto Alegre: Artmed.

Arrais, Alessandra da Rocha, \& Araujo, Tereza Cristina Cavalcanti Ferreira de. (2017). Depressão pós-parto: uma revisão sobre fatores de risco e de proteção. Psicologia, Saúde \& Doenças, 18(3), 828-845. https://dx.doi. org/10.15309/17psd180316

Arrais, Alessandra da Rocha, Araujo, Tereza Cristina Cavalcanti Ferreira de, \& Schiavo, Rafaela de Almeida. (2018). Fatores de Risco e Proteção Associados à Depressão Pós-Parto no Pré-Natal Psicológico. Psicologia: Ciência e Profissão, 38(4), 711-729. https://doi.org/10.1590/1982-3703003342016

Biscegli, T. S., Silva, G. S., Romualdo, P. F., Oliveira, M. S., Silva, B. R., \& Solim, F. (2017). Depressão pós-parto e tipo de parto: perfil de mulheres atendidas em um hospital-escola. CuidArte, Enferm, 11(1): 59-65. http:// ID: bde-31638

Cardoso, A. A., \& Vivian, A. G. (2017). Maternidade e suas vicissitudes: a importância do apoio social no desenvolvimento da díade mãe-bebê. Diaphora, 43-51.

Cunha, Ana Cristina Barros da, Santos, Carmelita, \& Gonçalves, Raquel Menezes. (2012). Concepções sobre maternidade, parto e amamentação em grupo de gestantes. Arquivos Brasileiros de Psicologia, 64(1), 139-155. Recuperado em 28 de abril de 2020, de http://pepsic.bvsalud.org/scielo. php?script $=$ sci_arttext\&ppi $=$ S1809-52672012000100011\&lng $=$ pt\&tlng $=$ pt

Ferreira, Cátia, Silva, Vanessa, Guerra, Cláudia, Silva, Ana Isabel, \& Rosário, Rafaela. (2018). Depressão pós-parto: deteção precoce e fatores associados. Acta Obstétrica e Ginecológica Portuguesa, 12(4), 262-267. Recuperado em 28 de abril de 2020, de http://www.scielo.mec.pt/scielo.php?script=sci_ arttext\&pip $=$ S1646-58302018000400003\&lng $=$ pt\&tlng=pt

Harrington, B. J., Hosseinipour, M. C., Maliwichi, M., Phulusa, J., Jumbe, A., Wallie, S., Pence, B. W. (2018). Prevalence and Incidence of Probable Perinatal Depression Among Women Enrolled in Option B+ Antenatal HIV Care in Malawi. J Affect Disord., 115-122. http:// doi: 10.1016/j. jad.2018.06.001

Hartmann, Juliana Mano, Mendoza-Sassi, Raul Andrés e Cesar, Juraci Almeida. (2017). Depressão entre puérperas: prevalência e fatores associados. Cadernos de Saúde Pública, 33 (9), e00094016. Epub 9 de outubro de 2017. https://doi.org/10.1590/0102-311x00094016

Hildebrandt, F. M. (2013). Depressão pós-parto: aspectos epidemiológicos e tratamento cognitivo-comportamental. Tese (doutorado). Programa de Pós Graduação em Psicologia, UFRJ, Rio de Janeiro, RJ.

Hollist, C. S., Falceto, O. G., Seibel, B. L., Springer, P. R., Nunes, N. A., Fernandes, C. L. C., \& Miller, R. B. (2016). Depressão pós-parto e satisfação conjugal: impacto longitudinal em uma amostra brasileira. Revista Brasileira De Medicina De Família E Comunidade, 11(38), 1-13. https://doi.org/10.5712/ rbmfc11(38)1044
Kliemann, A., Böing, E., \& Crepaldi, M. (2017). Fatores de risco para ansiedade e depressão na gestação: Revisão sistemática de artigos empíricos. Mudanças - Psicologia da Saúde, 25(2),69-76. http://dx.doi.org/10.15603/2176-1019/ mud.v25n2p69-76

Krob, Adriane Diehl, Godoy, Josehelen de, Leite, Keila Pamela, \& Mori, Samantha Gottardo. (2017). Depressão na gestação e no pós-parto e a responsividade materna nesse contexto. Revista Psicologia e Saúde, 9(3), 3-16. https://dx.doi.org/10.20435/pssa.v9i3.565

Lobato, Gustavo, Moraes, Claudia L, \& Reichenheim, Michael E. (2011). Magnitude da depressão pós-parto no Brasil: uma revisão sistemática. Revista Brasileira de Saúde Materno Infantil, 11(4), 369-379. https://doi.org/10.1590/ S1519-38292011000400003

Marques, L. d., Silva, W. R., Lima, V. P., Nunes, J. T., Ferreira, A. G., \& Fernandes, M. N. (2016). Saúde mental materna: rastreando os riscos causadores da depressão pós-parto. Journal Health NPEPS, 1(2): 145-159. http:// ID: biblio-1052312

Ministério da Saúde. (2009). Pesquisa Nacional de Demografia e Saúde da Criança e da Mulher - PNDS 2006. Brasilia: CEBRAP - Centro Brasileiro de Análise e Planejamento.

Ministério da Saúde - Depressão pós-parto: causas, sintomas, tratamento, diagnóstico e prevenção. (2019). Fonte: Ministério da Saúde: https://saude.gov.br/saude-de-a-z/saude-mental/depressao-pos-parto.

Oliveira, L. S., Matão, M. E., Diego, M. V., Resende, E. E., \& Cleusa, M. A. (2020). Depressão puerperal: Fatores associados e a frequência de risco através da escala de Edimburgo / Postpartum depression: Associated factors and a risk frequency for the Edinburgh scale. Brazilian Journal of Health Review, 1052-1062. https://doi.org/10.34119/bjhrv3n1-082

Oliveira, M. G., Teixeira, R. S., Costa, V. N., Alencar, P. H., Rodrigues, E. O., Lima, A. M., \& Chaves, A. F. (2019). Sentimentos de mulheres com depressão pós-parto frente ao aleitamento materno. Revista Oficial do Conselho Federal de Enfermagem, 88-92. https://doi.org/10.21675/2357-707X.2019. v10.n3.1702

OPAS/OMS. (30 de Março de 2017). Com depressão no topo da lista de causas de problemas de saúde, OMS lança a campanba "Vamos conversar". Fonte: Site da OPAS/OMS: https://www.paho.org/bra/index. php?option $=$ com_content\&view $=$ article\&id $=5385$ :com-depressao- no-topo-da-lista-de-causas-de-problemas-de-saude-oms-lanca-a-campanha-vamos-conversar\&Itemid $=839$

OPAS/OMS. (Março de 2018). Folha informativa - Depressão. Fonte: opas/oms: https:/ $/$ www.paho.org/bra/index.php?option $=$ com_content\&view $=$ articl e\&id $=5635$ :folha-informativa-depressao\&Itemid $=1095$

Santos, Iná S., Tavares, Beatriz Franck, Munhoz, Tiago N., Almeida, Laura Sigaran Pio de, Silva, Nathália Tessele Barreto, Tams, Bernardo Dias, Patella, André Machado, \& Matijasevich, Alicia. (2013). Sensibilidade e especificidade do Patient Health Questionnaire-9 (PHQ-9) entre adultos da população geral. Cadernos de Saúde Pública, 29(8), 1533-1543. https:// doi.org/10.1590/0102-311X00144612

Theme-Filha, M. M., Baldisserotto, M. L., Fraga, A. S., Ayers, S., Gama, S. G., \& Leal, M. (2016). Factors associated with unintended pregnancy in Brazil: cross-sectional results from the Birth in Brazil National Survey, 2011/2012. Reproductive Health, 236-265. https://doi.org/10.1186/s12978-016-0227-8

Yawn, B. P., Pace, W., Wollan, P. C., Bertram, S., Kurland, M., Graham, D., \& Dietrich, A. (Sep-Out de 2009). Concordance of Edinburgh Postnatal Depression Scale (EPDS) and Patient Health Questionnaire (PHQ-9) to assess increased risk of depression among postpartum women. J Am Board Fam Med, 483-491. https://doi.org/10.3122/jabfm.2009.05.080155

Submetido em: 29-4-2020

Aceito em: 20-12-2020 